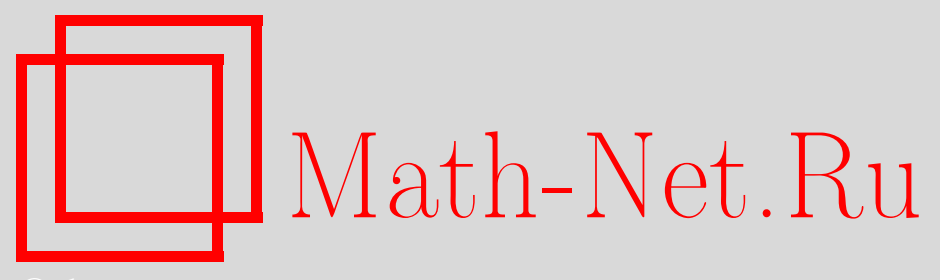

Н. П. Фадеев, Многочлены, ортогональные на двух симметричных промежутках, Матем. заметки, 1999, том 66, выпуск 5, 796-800

DOI: https://doi.org/10.4213/mzm1224

Использование Общероссийского математического портала Math-Net.Ru подразумевает, что вы прочитали и согласны с пользовательским соглашением http://www.mathnet.ru/rus/agreement

Параметры загрузки:

IP : 18.234 .156 .22

26 апреля 2023 г., $17: 57: 25$

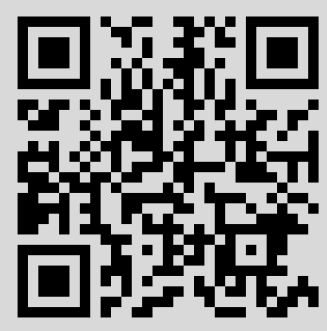




\section{МНОГОЧЛЕНЫ, ОРТОГОНАЛЬНЫЕ НА ДВУХ СИММЕТРИЧНЫХ ПРОМЕЖУТКАХ}

\section{Н. П. Фадеев}

Пусть $\varphi_{n}(x)(n=0,1,2, \ldots)$ - алгебраические многочлены, ортогональные относительно четной весовой функции $\rho(x)$ на множестве

$$
E=[-b,-a] \cup[a, b]
$$

где $0 \leqslant a<b \leqslant+\infty$ и

$$
\begin{gathered}
\frac{\rho^{\prime}(x)}{\rho(x)}=\frac{R_{4}(x)}{R_{5}(x)}, \quad V(x)=\frac{\rho(x)}{x^{3}} R_{5}(x), \quad V( \pm a)=V( \pm b)=0, \\
D(x)=\frac{R_{4}(x)+R_{5}^{\prime}(x)-\frac{2 R_{5}(x)}{x}}{x^{2}}
\end{gathered}
$$

- целая относительно $x$ функция, где $R_{k}(x)(k=4,5)$ - многочлен степени не выше $k$.

При любом натуральном $m$ функция

$$
\rho_{m}(x)=\left(\frac{R_{5}(x)}{x}\right)^{m} \rho(x)
$$

удовлетворяет на множестве (1) условиям (2), (3).

ЛЕмма 1. При любом натуральном $m$ многочлены $D^{m} \varphi_{2 n}(x)(n=m, m+1, \ldots)$, где

$$
D f=\frac{f_{x}^{\prime}}{x}
$$

ортогональны на мнохестве (1) относительно веса (4).

ДоКАЗАТЕЛЬство. Покажем, что в соответствии с определением ортогональных многочленов [1] при $m=1$ и $2 \leqslant k \leqslant 2 n-1$

$$
I_{k}=\int_{E} \rho_{1}(x)\left(D \varphi_{2 n}\right) x^{k-2} d x=0 .
$$

Действительно, пользуясь равенством (5) и после интегрирования по частям с условиями (2), (3), получим $I_{k}=0$. Для завершения доказательства леммы достаточно воспользоваться индукцией по $m$.

ТЕОРема 1. При любом $m=0,1,2, \ldots$ многочлены $D^{m} \varphi_{2 n}(x) \quad(n=m, m+1, \ldots)$ являются частными решениями дифференчиального уравнения

$$
R_{5}(x) y^{\prime \prime}+\left[R_{4}(x, m)+R_{5}^{\prime}(x)-\frac{3 R_{5}(x)}{x}\right] y^{\prime}-\gamma_{n, m} x^{3} y=0
$$

əəe

$$
R_{4}(x, m)=R_{4}+m\left(R_{5}^{\prime}-\frac{R_{5}}{x}\right), \quad \gamma_{n, m}=2 n\left[r_{0}^{(4)}(m)+(2 n+1) r_{0}^{(5)}\right]
$$


$r_{0}^{(4)}(m)$ - коэффициент при $x^{4}$ многочлена $R_{4}(x, m), r_{0}^{(4)}(0)=r_{0}^{(4)}, r_{0}^{(5)}$ - коэффициент при $x^{5}$ многочлена $R_{5}(x)$.

ДокАЗАТЕльСтво. Для случая $m=0$ теорема доказана в [2]. Доказательство общего случая сводится к замене веса $\rho(x)$ на вес $(4)$ и $R_{4}(x)$ на $R_{4}(x, m)$.

ТЕОРема 2. Если четная весовая функиия $\rho(x)$ удовлетворяет на множсестве (1) условиям (2), (3), то при $n=0,1,2, \ldots$

$$
\varphi_{2 n}(x)=k_{n} \frac{x}{\rho(x)} D^{n}\left[\rho(x) \frac{R_{5}^{n}(x)}{x^{n+1}}\right]
$$

әде $k_{n}$ - постоянный (стандартизирующий) мнохитель.

ДокАЗАТЕЛЬСтво. Покажем, что

$$
P_{2 n}(x)=\frac{x}{\rho(x)} D^{n}\left[\rho(x) \frac{R_{5}^{n}(x)}{x^{n+1}}\right]
$$

является четным многочленом степени $2 n$.

Действительно,

$$
D\left(\rho \frac{R_{5}^{n}}{x^{n+1}}\right)=\rho(x) \frac{R_{5}^{n-1}(x)}{x^{n}} Q_{2 n, 2}(x),
$$

где $Q_{2 n, 2}(x)$ - четный многочлен степени 2 .

Математической индукцией с применением условий (2), (3) легко показать, что

$$
D^{k}\left(\rho \frac{R_{5}^{n}}{x^{n+1}}\right)=\rho(x) \frac{R_{5}^{n-k}}{x^{n-k+1}} Q_{2 n, 2 k},
$$

где $Q_{2 n, 2 k}(x)$ - четный многочлен степени $2 k$.

При $k=n$ получим

$$
D^{n}\left(\rho \frac{R_{5}^{n}}{x^{n+1}}\right)=\frac{\rho}{x} Q_{2 n, 2 n}
$$

где $Q_{2 n, 2 n}(x)$ - четный многочлен степени $2 n$.

Учитьвая определение ортогонального многочлена, осталось показать, что при $k=0,1,2, \ldots, 2 n-1$

$$
I_{k}=\int_{E} \rho(x) P_{2 n}(x) x^{k} d x=0 .
$$

Применяя последовательно формулу интегрирования по частям и учитывая равенство (8) и условия $(2),(3)$, получим $I_{k}=0$. Откуда в силу известной теоремы единственности системы ортогональных многочленов [1] следует заключение теоремы 2.

Пусть

$$
\psi_{2 n}(x)=\frac{x}{\rho(x)} D^{n}\left[\rho(x) \frac{R_{5}^{n}}{x^{n+1}}\right] .
$$

Tеорема 3. При $n=0,1,2, \ldots$

$$
I=\int_{E} \rho \psi_{2 n}^{2} d x=(-1)^{n} 2^{n} n ! a_{2 n} \int_{E} \rho\left(\frac{R_{5}}{x}\right)^{n} d x,
$$

где $a_{2 n}$ - стариий коэффициент многочлена $\psi_{2 n}(x)$. 
ДОКАЗАТЕЛЬСТвО. Пусть

$$
U(x)=\rho(x) \frac{R_{5}^{n}(x)}{x^{n+1}}, \quad V(x)=\psi_{2 n}(x) .
$$

Тогда

$$
I=\left.\left(D^{n-1} U\right) V\right|_{\partial E}-\int_{E}\left(D^{n-1} U\right) V^{\prime} d x,
$$

где $\partial E=\{-b,-a, a, b\}$. В силу условий $(2),(3)$ и равенства (8) внеинтегральный член равен 0 .

Повторяя проделанные операции, получим

$$
I=(-1)^{n} \int_{E} x U D^{n} V d x
$$

Вместе с тем

$$
D^{n} V=D^{n} \psi_{2 n}=2^{n} n ! a_{2 n} .
$$

Для завершения доказательства теоремы осталось сопоставить равенства (10)-(13).

ТеОРема 4. Многочлены $\psi_{2 n}(x) \quad(n=0,1,2, \ldots)$ являются решениями интегрального уравнения Вольтерра второго рода

$$
\frac{\psi_{2 n}(x)}{U(x)}=c_{1} x^{3 / 2}+\frac{c_{2}}{\sqrt{x}}+\frac{1}{2 \sqrt{x}} \int_{x_{0}}^{x}\left(x^{2}-t^{2}\right) t^{3 / 2} R(t) \frac{\psi_{2 n}(x)}{U(t)} d t
$$

əəe

$$
U(x)=\exp \left(-\frac{1}{2} \int P(x) d x\right), \quad P(x)=\frac{x^{2} D(x)}{R_{5}(x)}-\frac{1}{x}, \quad D(x)=\frac{R_{4}+R_{5}^{\prime}-\frac{2 R_{5}}{x}}{x^{2}}
$$

$c_{1}, c_{2}$ - произвольные постоянные, $x_{0}$ - фиксированная точка,

$$
R(x)=\frac{1}{2}\left(\frac{x^{2} D(x)}{R_{5}(x)}\right)^{\prime}+\frac{1}{4}\left(\frac{x^{2} D(x)}{R_{5}(x)}\right)^{2}-\frac{x D(x)}{2 R_{5}(x)}-\frac{\gamma_{n} x^{3}}{R_{5}(x)} .
$$

ДокАЗАТЕльство. При $m=0$ дифференциальное уравнение (6) после деления его на $R_{5}(x)$ принимает вид

$$
y^{\prime \prime}+P(x) y^{\prime}+Q(x) y=0, \quad P(x)=\frac{x^{2} D(x)}{R_{5}(x)}-\frac{1}{x}, \quad Q(x)=-\frac{\gamma_{n} x^{3}}{R_{5}(x)},
$$

где первая дробь в выражении $P(x)$ не выделяет слагаемого, содержащего $x^{-1}$.

Известно [3], что в уравнении (15) подстановкой

$$
y(x)=U(x) z(x)
$$

можно уничтожить член, содержащий $y^{\prime}$. Тогда

$$
U=\exp \left(-\frac{1}{2} \int P(x) d x\right), \quad z^{\prime \prime}+\left[-\frac{1}{2} P^{\prime}(x)-\frac{1}{4} P^{2}(x)+Q(x)\right] z=0 .
$$

В нашем случае

$$
U(x)=\exp \left[-\frac{1}{2} \int\left(\frac{x^{2} D(x)}{R_{5}(x)}-\frac{1}{x}\right) d x\right], \quad x^{2} z^{\prime \prime}-\left(\frac{3}{4}+x^{2} R(x)\right) z=0,
$$

где $R(x)$ имеет вид (14).

Последнее дифференциальное уравнение можно формально записать как неоднородное. 
Тогда, как известно [3], если $z_{1}(x), z_{2}(x)$ - фундаментальная система частных решений соответствующего однородного уравнения, то общее решение уравнения (16) имеет вид

$$
z=C_{1} z_{1}(x)+C_{2} z_{2}(x)+\int_{x_{0}}^{x} \frac{z_{1}(x) z_{2}(t)-z_{1}(t) z_{2}(x)}{z_{1}^{\prime}(t) z_{2}(t)-z_{1}(t) z_{2}^{\prime}(t)} z(t) t^{2} R(t) d t .
$$

Учитывая обозначения, введенные в начале доказательства, и то, что в нашем случае

$$
z_{1}=x^{3 / 2}, \quad z_{2}=x^{-1 / 2},
$$

получим интегральное уравнение теоремы 4 .

Условиям (2), (3) удовлетворяет на множестве

$$
E=[-1,-\gamma] \cup[\gamma, 1]
$$

весовая функция

$$
\rho(x, \alpha, \beta, \gamma)=|x|\left(1-x^{2}\right)^{\alpha}\left(x^{2}-\gamma^{2}\right)^{\beta},
$$

где $\alpha>-1, \beta>-1,0 \leqslant \gamma<1$.

В соответствии с теоремой 1 многочлены $\varphi_{2 n}(x, \alpha, \beta, \gamma)(n=0,1,2, \ldots)$ являются частными решениями дифференциаљного уравнения (6) $(m=0)$.

TЕорема 5. Eсли $q=\max \{\alpha, \beta\} \geqslant-1 / 2$, то максимум абсолютного значения многочлена $\widetilde{\varphi}_{2 n}(x, \alpha, \beta, \gamma)$ достигается на множестве $E$ в одной из его граничных точек.

ДокАЗАТЕЛЬСтво. Положим $y=\widetilde{\varphi}_{2 n}(x, \alpha, \beta, \gamma)$. Так как $y(x)$ - функция четная, достаточно изучить ее поведение на отрезке $[\gamma, 1]$. Рассмотрим функцию [4]

$$
U=y^{2}-\frac{R_{5}(x)}{\gamma x^{3}}\left(y^{\prime}\right)^{2} .
$$

Дифференцируя это равенство, получим

$$
U^{\prime}=2 y y^{\prime}-\frac{R_{5}^{\prime}-3 x^{-1} R_{5}}{\gamma_{n} x^{3}}\left(y^{\prime}\right)^{2}-\frac{R_{5}}{\gamma_{n} x^{3}} 2 y^{\prime} y^{\prime \prime} .
$$

Так как многочлен $\widetilde{\varphi}_{2 n}(x, \alpha, \beta, \gamma)$ является решением дифференциального уравнения (6), то

$$
U^{\prime}=\frac{2 R_{4}+R_{5}^{\prime}-3 x^{-1} R_{5}}{\gamma_{n} x^{3}}\left(y^{\prime}\right)^{2}
$$

или (см. условие (2) и вес (18))

$$
U^{\prime}=\frac{2(\alpha+\beta+1) x^{2}-(2 \alpha+1) \gamma^{2}-2 \beta-1}{2 n(\alpha+\beta+n+1) x}\left(y^{\prime}\right)^{2} .
$$

Рассмотрим пока случай, когда

$$
\alpha>-\frac{1}{2}, \quad \beta>-\frac{1}{2} .
$$

Тогда последнее равенство можно записать в виде

$$
U^{\prime}=\frac{\alpha+\beta+1}{n(\alpha+\beta+n+1) x}\left(x^{2}-x_{0}^{2}\right)\left(y^{\prime}\right)^{2},
$$

где

$$
x_{0}=\left(\frac{(2 \alpha+1) \gamma^{2}+2 \beta+1}{2(\alpha+\beta+n+1)}\right)^{1 / 2} .
$$

Точка $x_{0}$ находится внутри интервала $] \gamma, 1$, так как $0<\gamma<1$. Из равенства (21) видно, что производная $U^{\prime}(x)$ меняет знак в точке $x_{0}$, причем на интервале $] \gamma, x_{0}$ [ она отрицательна, а на 
интервале $] x_{0}, 1[$ - положительна. Следовательно, функция $U(x)$, определяемая равенством (19), убьвает на интервале $] \gamma, x_{0}$ [и возрастает на интервале $] x_{0}, 1[$. Из равенства (19) следует, что функция $U(x)$ положительна в интервале $] \gamma, 1[$. Поэтому

$$
U(x) \leqslant U(\gamma), \quad x \in] \gamma, x_{0}[; \quad U(x) \leqslant U(1), \quad x \in] x_{0}, 1[.
$$

Из равенства $(19)\left(y=\widetilde{\varphi}_{2 n}(x, \alpha, \beta, \gamma)\right)$ следует, что

$$
\widetilde{\varphi}_{2 n}^{2}(x, \alpha, \beta, \gamma) \leqslant U(x), \quad \widetilde{\varphi}_{2 n}^{2}(\gamma, \alpha, \beta, \gamma)=U(\gamma), \quad \widetilde{\varphi}_{2 n}^{2}(1, \alpha, \beta, \gamma)=U(1) .
$$

Из последних пяти соотношений видим, что

$$
\begin{array}{ll}
\left|\widetilde{\varphi}_{2 n}(x, \alpha, \beta, \gamma)\right| \leqslant\left|\widetilde{\varphi}_{2 n}(x, \alpha, \beta, \gamma)\right|, & x \in\left[\gamma, x_{0}\right], \\
\left|\widetilde{\varphi}_{2 n}(x, \alpha, \beta, \gamma)\right| \leqslant\left|\widetilde{\varphi}_{2 n}(x, \alpha, \beta, \gamma)\right|, & x \in\left[x_{0}, 1\right] .
\end{array}
$$

При $\alpha \geqslant-1 / 2,-1<\beta \leqslant-1 / 2$ и при $-1<\alpha \leqslant-1 / 2, \beta \geqslant-1 / 2$ рассуждения аналогичны. Приведенные рассуждения теряют смысл, если $\alpha=\beta=-1 / 2$. Однако нетрудно показать, что в этом случае многочлены $\widetilde{\varphi}_{2 n}(x, \alpha, \beta, \gamma)(n=0,1,2, \ldots)$ равномерно ограничены.

\section{СПИСОК ЦИТИРОВАННОЙ ЛИТЕРАТУРЫ}

1. Суетин П. К. Классические ортогональные многочлены. М.: Наука, 1979. 2. Фадеев Н.П. // Изв. вузов. Матем. 1979. № 5(168). С. 99-103. 3. Сегё Г. Ортогональные многочлены. М.: Физматгиз, 1962. 4. Сонин Н. Я. Исследования о цилиндрических функциях и специальных полиномах. М.: Гостехиздат, 1954.

Мордовский государственный педагогический институт 